\title{
Effect of Moisture Absorption on Hardness of Surface Treated Luffa Cylindrica /Polyster Composite
}

\author{
J. Benny, D. N. Arun selvan, G. Kalusuraman, I. Siva,
}

\begin{abstract}
The natural fibers have been received much more attention in research due the better compatibility, high strength, easy availability etc. The work addresses the moisture absorption on surface treated luffa fiber reinforced polyester composite. The fibers are treated with $\mathrm{NAOH}$ solution. The luffa fiber reinforced polyester composite were prepared by the compression moulding technique with optimum pressure of 17MPa. The composites were prepared for $50 \%$ fiber loading condition. The prepared composites are subjected to moisture absorption test for $1 \mathrm{hr}, 5 \mathrm{hr}$ and 10hrs. The result shows that the treated composite possesses the high hardness than the untreated composites.
\end{abstract}

Keywords : Luffa, Polyester, surface treatment.

\section{INTRODUCTION}

The increasing demand of natural fiber as a reinforcement material in the research and industry has been much more received. The natural fiber has turned in to reasonable substitute in synthetic or manmade fibers as a reinforcement material due to their enormous pros such as, low density, low cost, higher strength to weight ratio, recyclability and biodegradability when compared to synthetic fibers [ 1-2]. The cost of manufacturing the natural fiber reinforced composite with thermosetting polymer is quite low. The increase in moisture abortion is occurred in increasing the fiber lading. Thamos et al [3] analyzed that the relationship between the fiber loading and moisture absorption of pine apple-leaf fibre reinforced low density polyethylene (LDPE) composites. and also reported that the moisture content is increased as the fiber loading increased, Pothan [4]et all studied that hybridization and chemical modification effects on banana fiber/polyester composite and chemical modification was found to have emotional impact for the water absorption in the composites. Rao et al.[5] presented a comprehensive moisture absorption analysis in a jute/epoxy composite. Dhakal et al [6] studied that the effect of water update on mechanical properties of the hump / polyester composite and also reported that mechanical

Revised Manuscript Received on December 16, 2019.

* Correspondence Author

J. Benny*, Departmen of Mechanical Engineering, Kalasalingam Academy of Research and Education, Krishnankoil-626 126, India, Email: jbenny290799@gmail.com

DN. Arunselvan, Department of Mechanical Engineering, Kalasalingam Academy of Research and Education, Krishnankoil-626 126, India, Email: arunselvan29@gmail.com

N G. Kalusuraman*, Faculty of Mechanical Engineering, Kalasalingam Academy of Research and Education, Krishnankoil-626 126, India, Email: kalusunrk@gmail.com

I, Siva , Faculty of Mechanical Engineering, Kalasalingam Academy of Research and Education, Krishnankoil-626 126, India, Email: isiva@klu.com properties were decreased with increase in percentage of moisture content. Tanobe et al. [7] reported that the chemical modification of luffa fibers can improve their physical properties as to bring the better compatibility with composites. Ghali et al. [8] reported that alkali treatment such as sodium hydroxide and hydrogen peroxide can increase their crystallinity amount by bringing out the most amorphous materials from the luffa fibers. The so many studies have been carried out on the natural fiber reinforced composite by many researches and these moisture update would be done by the utilization of coupling agents and chemical treatment [9-11]

The objective of study that the effect of chemical treatment on hardness of luffa cylindrica polyester composite.

\section{MATERIALS AND METHODS}

\section{A. Materials used}

Luffa fruit fibers were supplied form the Rajapalayam, Tamilnadu, India. The figure 1 shows the image of luffa finer. In this work unsaturated polyester resin was utilized as matix. In this work, the initiator as Methyl ethyl ketone peroxide and accelerator as cobalt naphthenate were used at $1.5 \%$ each for the fabrication of the composite. $\mathrm{NaOH}$, procured from the Modern Scientifics, Madurai, Tamilnadu was utilized to modify the surface of the fiber.

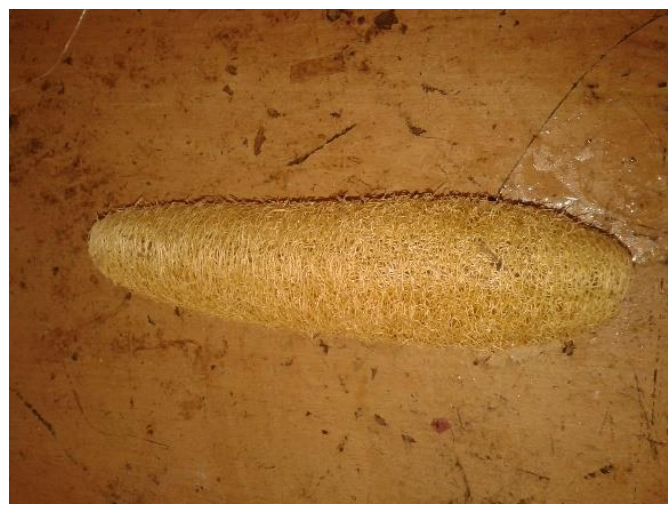

Fig.1. The photo snap of luffa fruit fiber 


\section{B. NaOH treatment}

The luffa fiber is treated with Sodium hydroxide $(\mathrm{NaOH})$ to remove the impurities present in it. Sodium hydroxide is in the form of white pellets, $40 \mathrm{gm}$ of sodium hydroxide is taken to dissolve in 1litre of distilled water. The luffa fiber is kept immersed for an hour in the in sodium hydroxide solution, then it was washed with distilled water thoroughly. Then it is dried to remove all its moisture content and used for fabrication of composite

\section{Composite fabrication}

The luffa fiber were stacked on the mold cavity as per requirement. Then the mold is pre compressed in the compression molding machine. Then the wax is applied in the mold cavity. then pre compressed fiber were placed in the mold. Then required unsaturated polyester resin along with catalyst and accelerated with $1.5 \%$ each were poured on the fiber in the mold. The mold is compressed with help of compression molding machine with the optimum pressure 17 Mpa. Then sample was taken from the mold after the $24 \mathrm{hr}$ curing. The required $50 \%$ fiber loading composite were cut for hardness testing. Fig 2. Shows that snapshot of prepared composite.

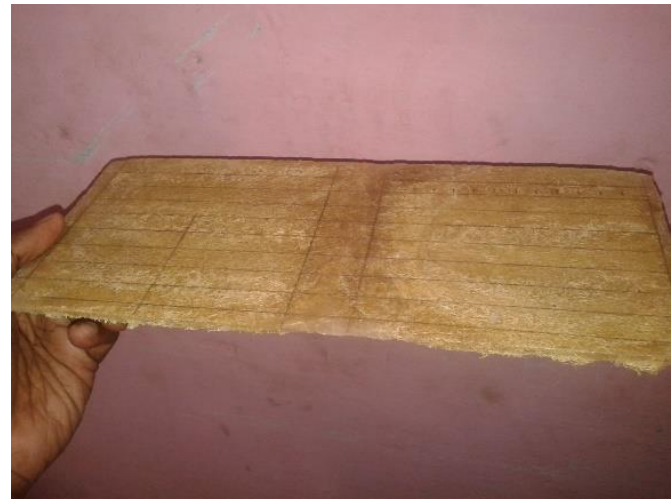

Fig 2. Snap shot of prepared composite

\section{MECHANICL TESTING}

\section{A. Hardness testing}

Shore D hardness test was performed using a durometer as per ASTM D2240. Initially cleaned and dried samples were pinged with the indenter of the Shore D hardness tester. Based on the depth produced, direct measure of hardness

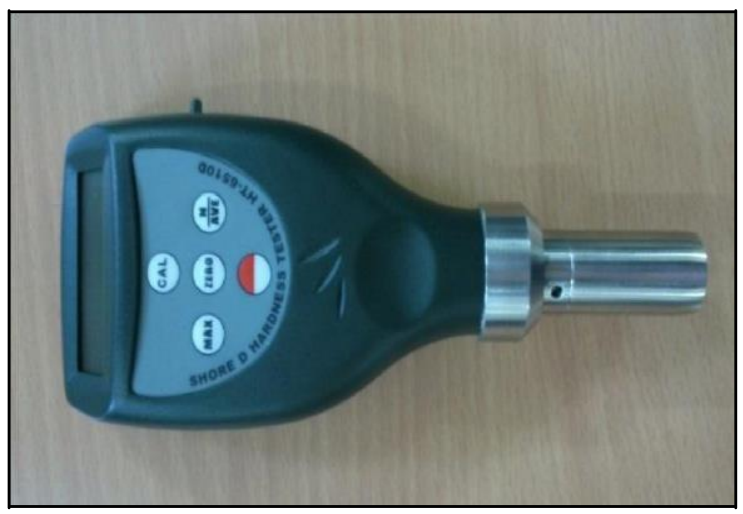

Fig.3. Hardness testing instrument

\section{B. Moisture absorption}

The samples (Untreated and $\mathrm{NaOH}$ ) Treated) were immersed in the sea water for $1 \mathrm{hr}, 5 \mathrm{hrs}$ and $10 \mathrm{hrs}$ separately. After that samples were subjected in to the hardness test.

\section{RESULTS AND DISCUSSION}

The fig 4. Shows that the effect of moisture absorption on the hardness of the prepared composite. The two kind of composite prepared from the compression molding machine. i.e untreated composite and treated composite. Shore D instrument is used to measure the hardness of the moisture absorbed composites. The samples were immersed in the sea water for $1 \mathrm{hr}, 5 \mathrm{hrs}$ and $10 \mathrm{hrs}$. It is observed that the treated composites is showing the higher hardness than the untreated composite for all kind of the testing condition. Form the $\mathrm{NaOH}$, the fiber surface roughness was increased. Due to the increasing the surface roughness the better interlocking between the finer and matrix can be achieved

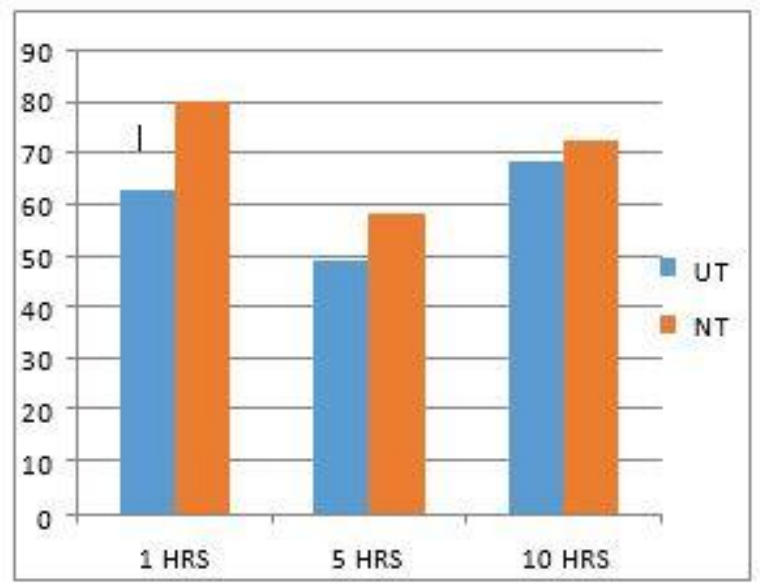

Fig 4. Effect of moisture absorption on hardness of treated and untreated composites,

\section{CONCLUSION}

The following conclusion were made from this work - The luffa cylindrica/polyester composites were fabricated using the compression molding machine successfully

- Treated composite possess high hardness compared to untreated composite

\section{REFERENCES}

1. Alejandro Hernández, Rossette Zdzislaw Mazur C, Alain Demeulenaere, J.A.Roque Lopez Hernández "The effect of start-up cycle in ceramic coating used as thermal barrier for a gas turbine bucket," Applied Thermal Engineering, Vol 29, pp 3056-3065(2009)

2. L. Wang, Y. Wang, X.G. Sun, J.Q. He, Z.Y. Pan, C.H. Wang, "A novel structure design towards extremely low thermal conductivity for thermal barrier coatings - Experimental and mathematical study, Materials and Design", pp505 -517 (2012)

3. Lisa Pin, Florence Ansart, Jean-Pierre Bonino, Yannick Le Maoult, Vanessa Vidal, Philippe Lours, "Processing, repairing and cyclic oxidation behavior of sol-gel thermal barrier coatings", Surface and Coatings Technology, Vol. 206, pp 1609 $1614(2011)$ 
4. L. Wang, Y. Wang, X.G. Sun, J.Q. He, Z.Y. Pan, Y. Zhou, P.L. Wu, "Influence of pores on the thermal insulation behavior of thermal barrier coatings prepared by atmospheric plasma spray", Materials and Design, Vol.32, pp 36-47 (2011)

5. Shen Wei, Wang Fu-chi, Fan Qun-Bo, Ma Zhuang, "Effects of defects on the effective thermal conductivity of thermal barrier coatings", Applied Mathematical Modelling, Vol.36, pp 1995-2002 (2012)

6. Lorenzo Malavasi, Maria Cristina Mozzatib, Stefano Polizzi, Carlo Bruno Azzoni and Giorgio Flor, "Nanosized Sodium-Doped Lanthanum Manganites: Role of the Synthetic Route on their Physical Properties" Chemistry of Materials, Vol.15, pp (2003)

7. Rodica Rogojan, Ecaterina Andronescu, Cristina, Ghiţulică and Bogdan Stefan Vasile, "Synthesis and Characterization of Alumina Nano-Powder Obtained By Sol-Gel Method", UPB Scientific Bulletin, Series B: Chemistry and Materials Science,Vol.73, pp $67-$ 76 (2011)

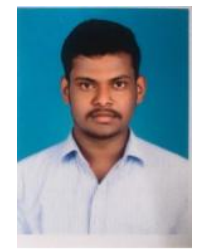

\section{AUTHORS PROFILE}

J.Benny is currently pursuing UG degreein the Department of Mechanical Engineering, Kalasalingam Academy of Research and Education, Anand nagar, Krishnankoil-626126,India

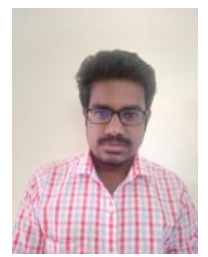

DN.arun selvan is currently pursuing UG degreein the Department of Mechanical Engineering, Kalasalingam Academy of Research and Education, Anand nagar, Krishnankoil-626126,India

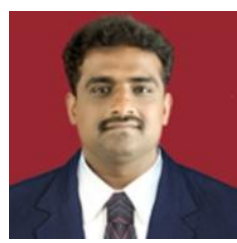

Dr. G. Kalusuraman is currently working as Associate Professor in the Department of Mechanical Engineering, Kalasalingam Academy of Research and Education, Anand nagar, Krishnankoil-626126,India $\mathrm{He}$ has 10 years teaching as well as research experience. He is a reviewer for two reputed journals $\mathrm{He}$ is a Member of Indian Society for Technical Education .His area of interests are polymer

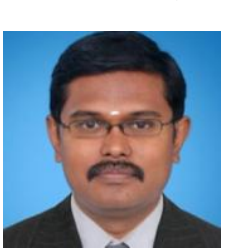
composite, materials characterization, tribology.

Dr. I. Siva is currently working as Professor in the Department of Mechanical Engineering, Kalasalingam Academy of Research and Education, Anand nagar, Krishnankoil-626126,India He has 15 years teaching as well as research experience. He is a reviewer more than 10 reputed journals $\mathrm{He}$ is a Member of Indian Society for Technical Education .His area of interests are polymer composite, materials characterization, tribology. 